\title{
Carcinogenic Properties of Aflatoxins in Maize Production and Recent Technologies Involved
}

\author{
Mehmood N ${ }^{1}$, Sohail F², Sohail S ${ }^{3 *}$, Aslam $\mathbf{M}^{4}$, Ali \\ $\mathrm{M}^{5}$, Ijaz N $\mathbf{N}^{3}$, Aslam $\mathrm{T}^{\mathbf{1}}$ and Rana $\mathrm{H}^{6}$ \\ ${ }^{1}$ MS Biochemistry, University of Central Punjab, Pakistan \\ ${ }^{2}$ BS Microbiology, Government College University, \\ Pakistan \\ ${ }^{3}$ MS Microbiology, University of Central Punjab, Pakistan \\ ${ }^{4}$ MS Biotechnology, Government College University, \\ Pakistan \\ ${ }^{5}$ MS Biotechnology, University of Central Punjab, \\ Pakistan \\ ${ }^{6}$ MBBS, Allama Iqbal Medical College, Pakistan \\ *Corresponding author: Shehreen Sohail, MS \\ Biochemistry, University of Central Punjab, Pakistan
}

Received: August 16, 2021; Accepted: September 16 , 2021; Published: September 23, 2021

\begin{abstract}
Aflatoxins are the carcinogenic, mutagenic and highly toxic secondary metabolite, which is produced by two species of fungus such as Aspergillus flavus and Aspergillus parasiticus mostly. These two types of fungus contaminate the cereals crops such as maize, cotton, rice and spices. These are naturally occurring toxins all over the world. There are 20 different types of aflatoxins but out of six have significance importance for instance, AFB1, AFB2, AFG1, AFG2, AFM1 and AFM2. Most commonly, AFB1 and AFB2 are present in the maize crops and M1 and M2 present in the milk and dairy products. Even the small amounts of aflatoxins, which may cause the harmful effects in human and liver stocks. They cause many acute, subacute and chronic diseases such as hepatic carcinoma in human. Aflatoxins can pose a significant economic burden. It caused up to $25-30 \%$ approximately destroy the crops all over. Aflatoxicosis detection from the humans as well as animals is very tough because variation may occur, or sign and symptoms may be change due to the suppressor of immune system that cause many chronic diseases. These aflatoxins are determined and analyzed by the many techniques such as chromatographic techniques mostly HPLC, immunoassays, fluorescence spectroscopy and many biosensors which are very essential to check the quality, quantity and concentration of aflatoxins present in food and feeds. So that, there are many methods to limit the growth of aflatoxins from the grain in pre-harvest, during harvest and post-harvest conditions or also control the environmental conditions and managed the proper storages of the grains that may diminish the aflatoxins levels. Furthermore, they are many techniques and biological control methods are used to control the growth and activities of fungus species that cause the toxicity in food and feeds and protect the grains for the uses of human and animals.
\end{abstract}

Keywords: Aflatoxins; Aspergillus flavus; Aspergillus parasiticus

\section{Introduction}

Aflatoxins are the set of secondary metabolites, which are very poisonous, and have able to cause the cancer. They are produced by the toxic fungus specie Aspergillus for instance Aspergillus flavus, Aspergillus parasiticus, and Aspergillus nomius [1]. Aspergillus flavus is the main type of aspergillus species and have the importance in toxicology because this type of fungus produces aflatoxins and other carcinogenic chemicals such as aspergillic acids, cyclopiazonic acids and helvellic acid etc [2-3]. Aspergillus flavus and Aspergillus parasiticus mostly involved into the production of aflatoxins in food products for instance groundnut, peanuts, maize and other cereals. In polyketide pathway, aflatoxins are produced [4]. Aflatoxins are naturally occurring carcinogens which may cause the hepatocellular carcinoma because of the over consumption of aflatoxins because they suppress the immune system in human and livestock's [57]. Aflatoxins firstly isolated from the X Turkey Disease such as hepatic necrosis in 1960. There are 20 different types of aflatoxins involved into the agriculture foodstuff but six out of 18 have more dominant in food items named as B1, B2, G1, G2, M1 and M2 [8-10]. In all of them, aflatoxins B1 are the most significant and extensively found into the food and feed [11,12]. The Food and Agricultural Organization (FAO) given the report that $25 \%$ food contaminated by the mycotoxins. Approximately 70-75\% B1 aflatoxins are present into the agricultural feed [13]. Other types of aflatoxins M1 and M2 are related with the milk and these M1 and M2 type of the aflatoxins are the hydroxylate goods of the B1 and B2 aflatoxins. These are the contamination, which are present into the cow milk. During the milk processing, M1 and M2 are remain present into the milk [14]. Most important contamination occur by the Aspergillus fungus and its fungal infection on the crops occur in the humidity environment and damage the crop in the drought condition. These aflatoxins are chemically nature and cause many abnormalities, liver carcinoma and other mutations. It may be lethal when the dose of aflatoxins is higher than its requirements [15]. There are many acute and chronic diseases, which are caused by the exposure of the aflatoxins. Dose depends on the diseases either low dose or high dose. Exposure of low dose cause acute disease and the exposure in low dose for a long time may cause the serious effect on humans, they may cause the chronic disease. Exposure in high dose directly cause the chronic illnesses such as tumors.

Exposure in high dose directly cause the chronic illnesses such as tumors. In chronic exposure of the diseases, there are many severe diseases such as teratogenicity, mutagenicity, cytotoxicity, immunosuppression and estrogenic effects in humans and other 
mammals [16]. Aflatoxins caused nutritional illnesses such as growth faltering and these aflatoxins involved into the absorption of the micronutrients such as vitamins, iron and zinc etc. It is interfering into the formation of proteins, enzymes synthesis and metabolic activities by using the enzymes $[17,18]$. In domestic animals, aflatoxins may interfere into the feed of animals. Animal feed have some amounts of aflatoxins, animals eat the food and feed and caused minimum production of milk and reproduction, easily assessable to the diseases and reduce good quality meat productions [19]. The objective is aflatoxins, history of aflatoxins, types and properties, sources for the isolation of aflatoxins and disease related to the contamination and also metabolites of aflatoxin, treatment and biological control by microorganism such as bacteria and fungus.

\section{Fungal Type Related to Aflatoxins}

Aflatoxins mean the (A-Flavus toxins) and it is most important type of the mycotoxins. Mycotoxins are the toxic compounds that are naturally produce by the different types of molds or fungus. Aflatoxins related the genera of Aspergillus specie of the fungus. First time, it is isolated from the $\mathrm{X}$ Turkey disease such as hepatic necrosis in 1960 [20]. These aflatoxins are obtained after the X Turkey disease and aflatoxins and its metabolites are characterized from feed, which is contaminated by molds. The most important types of aflatoxins are B1, B2, G1 and G2. These types of aflatoxins can be determined on the basis of blue and green fluorescence under the ultraviolet light and it is also characterized by the thin layer chromatography during the silica gel chromatographic mobility. On the other hand, there are many different types of aflatoxins other than four major types are P1, Q1, B2a, and G2a. These are mammalian biotransformation products, which are obtained from the major metabolites [21-23]. For instance, the cow milk has the aflatoxins M1 which is hydroxylated derivatives of the $\mathrm{B} 1$ aflatoxin, and it is obtained from the feeds of animals, which is contaminated by the molds [24]. There are many acute and toxic effects on the animals, rats, rabbits, trout, duckling and the other vertebrates as well as the human are most prone to the aflatoxin effects. So that, the effects of toxins demonstrate by the dose. The toxicity depends upon the dose. The lower amount of dose but for a long period time exposure may cause the tumors (abnormal growth of the cells), most susceptible in liver. Trout and rats, which are most prone towards the carcinogenic effect of aflatoxins B1. So that, aflatoxins B1 is most familiar to cause the cancer in living organism and other vertebrates (Rushing and Selim 2019). So that, aflatoxins have the oxygenated poly-substitution coumarins and slightly differ from each other and these are produced by Aspergillus species of fungus. Aflatoxins are entered in the food chain of living organism when the molds grow on the food and feeds. There are mainly two species of molds, which are mostly prone to aflatoxin contamination, such as Aspergillus flavus and Aspergillus parasiticus. Another specie which is near to the Aspergillus flavus named as Aspergillus nomius also have the aflatoxigenic properties. It is reported in 1987 [25]. But in present, there are many other types of fungi which are susceptible to the production of aflatoxins, for instance Aspergillus tamarii [26], Aspergillus pseudotamarii [27], Aspergillus bombycis [28] and Emericella astellata [29]. In comparison of other species types, Aspergillus flavus and Aspergillus parasiticus are most important types which are used to determine the level of aflatoxins in food and feeds, other types of fungus are used in rare studies. The biosynthesized amount of the aflatoxins will change due to the two reasons, firstly the strain of fungi, which is used and second is growth conditions.

\section{Structures of Aflatoxins}

There are 20 different types of the aflatoxins, six are most important and widely used in food and feed. Aflatoxins B (AFB1 \& AFB2), Aflatoxins G (AFG1 \& AFG2) and Aflatoxins M (AFM1 \& AFM2) [30].

Aflatoxins B type are pentanone derivatives, displays the blue fluorescence under the ultraviolet light but in aflatoxins $G$ type are six membrane lactone and they give the green-yellow fluorescence on the thin layer chromatography film $[32,33]$. AFB1 and AFG1 are major aflatoxins and other are derived from them such as AFB2 and AFG2 and these are dihydroxy derivatives of the AFB1 and AFG1 and the other type of the aflatoxins are present in food and feed when the AFB1 is present in it [34]. The other type of the aflatoxin is aflatoxin M. It is a derived from the AFB1 and it is present into the dairy products such as milk of animals and it give the blue-violet fluorescence in thin layer chromatography $[35,36]$. Aflatoxins are the secondary metabolites and carcinogenic property and it is produced by the soilborne fungus named as Aspergillus flavus, Aspergillus parasiticus, Aspergillus tamarii and Aspergillus nomius. B-types aflatoxins are produced by most of the fungus dominated such as Aspergillus parasiticus, Aspergillus nomius, Aspergillus toxicarius and Aspergillus arachidicola and these also involved into the production of G-types aflatoxins [37]. Aspergillus flavus and Aspergillus parasiticus are popular to the development of AFM1, AFM2, AFB2A, AFG2A and other AFGM1.

\section{Crops Contaminated by Aflatoxins}

The major foods for the human consumption worldwide are cereals and cereal based products [38]. In the category of cereals and cereals-based products such as rice and maize are most common crops contaminated by the aflatoxins produce by the fungus species naturally because of change in the agricultural conditions, these aflatoxins are produced in the post and pre-harvest conditions of the crops $[39,40]$. Many reports give the results that rice are most important cereals which contaminated by the aflatoxins as compare to the other cereals crops, it is because of moisture content present during the drying process involved in the contamination and fungus growth increased and these fungi may cause the discoloration of the rice grains and destroy the quality of grains [41].

On the other hand, maize or corn is also contaminated by the aflatoxins. It is staple food in different countries, maize have greater moisture content then the other cereals and fungus grow easily in maize because of moisture. Aspergillus Flavus species may attack on the maize crop and it damage whole grains of maize. Fungal growth in the staple food depends upon the temperature, moisture content, physical and chemical condition of soil and the storage condition of the staple cereals [42].

Like other cereals, maize is the growing above the ground, and it is contaminated by aflatoxins and airborne contiguous fungi spores during plant developmental process, this condition is known as preharvest, during harvesting and after the harvesting the other process is handling, known as postharvest. Basic reason to contamination occurred is improper handling or activities during 

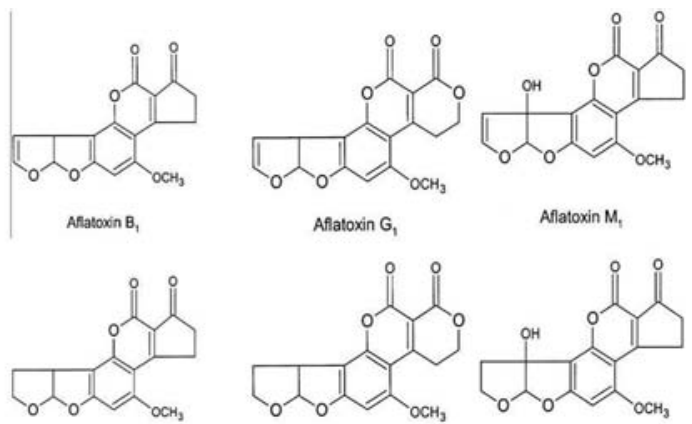

Afatoxin $G_{1}$

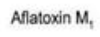

Aflatoxin $\mathrm{B}_{2}$
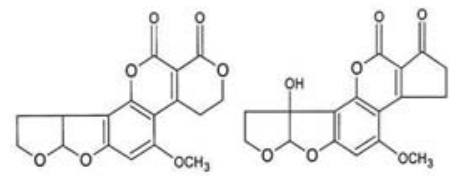

Aflatoxin $G_{2}$

Aflatoxin $\mathrm{M}_{2}$

Chemical structure of aflatoxin $B\left(\mathrm{AFB}_{1}\right.$ and $\left.\mathrm{AFB} \mathrm{B}_{2}\right)$, aflatoxin $\mathrm{G}\left(\mathrm{AFG}_{1}\right.$ and $\left.\mathrm{AFG}_{2}\right)$, and aflatoxin $\mathrm{M}\left(\mathrm{AFM}_{1}\right.$ and $\left.\mathrm{AFM}_{2}\right)$.

Figure 1: Aflatoxins and its types. AFB1, AFB2, AFG1, AFG2, AFM1 and AFM2 [31].

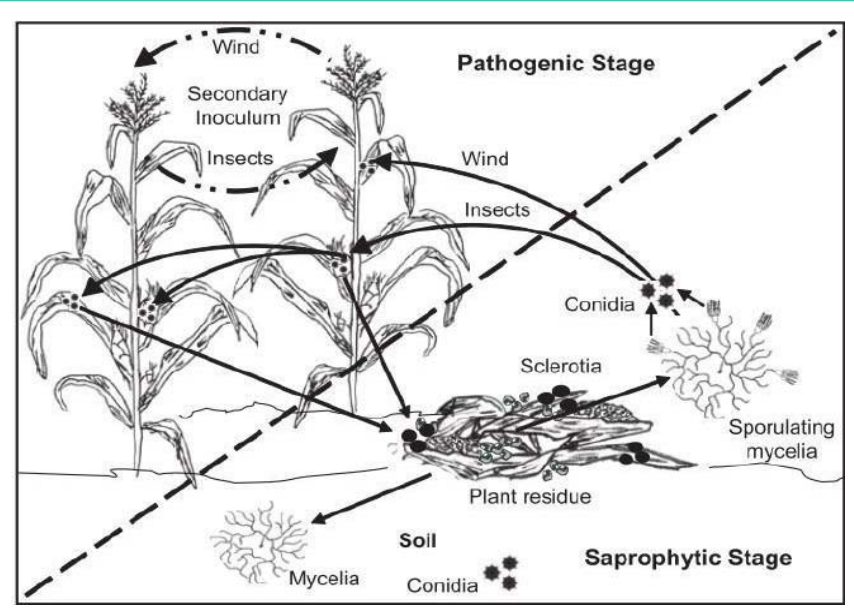

Figure 2: Life cycle of Aspergillus flavus on maize at Saprophytic stage.

the cultivation process that carried the fungus spores to the storage stage and the postharvest method. Aflatoxin's contamination occurs due to the improper storage of the cereals. Staple foods and other cereals not contaminated just by aflatoxins but there are many other mycotoxins and fumonisin, which are dangerous for the human as well as animals. The agricultural or in rural commodities which are most susceptible to the aflatoxin contaminations in maize and maize products, animal feeds and most the nut tress [43]. In term of international market and the financial development country which are involved in the trade market for example easily involve in the import and export are more prone to discuss the aflatoxin toxicity. To overcome these challenges many countries have set most extreme satisfactory levels of aflatoxins in food, food items and animal feeds $[44,45]$. Aflatoxin's contamination is varied due to the geological areas, agrarian and agronomic practices, storage condition of crops and more imperatively preparing of food materials under suitable temperature and humidity conditions [46].

\section{Life Cycle of the Fungus in Maize}

Aspergillus flavus is a type of saprophytic fungus that survives on the dead tissues of plants and it is also act as weak and resourceful pathogen [47]. This type of fungus needs the source such as moisture content, and it is easily produced and spread in all fields and grains.
Repeatedly, grow the crop of maize and it is also mingled with the crop of groundnuts and cottons, conidia from the sporogenic sclerotia are the first and primary source of the Aspergillus flavus species inocula. Conidia adjoining to the developing maize cobs that is germinated in the ground, carbon and nitrogen substrate released into the injured cobs of maize and it is result obtain by the colonization of the maize cobs. The released of the spores on the plant residues need the hot or warm humid favorable conditions. These spores are spread through the wind in the whole field [48]. Conidia which is present on the upper part of the plant residues and it bind with the body of birds and physically by using the plant body, it spread through all field. So that the improper or immature crop easily effected then the proper or mature maize field.

In the Figure 2, pathogenic stage of the fungus is shown. Conidia, mycelia and sclerotia are present in the plant residues. Through the insects and wind, conidia are spread in the cobs of maize and it destroy all the field.

\section{Disease Linked to the Aflatoxins}

The toxicity of aflatoxins has greater risk to cause the acute and chronic disease, which may cause the serious illness and even death, in the developing countries for example in Africa. Due to the aflatoxin contamination, there are many diseases occurred such as hepatocellular carcinoma in the presence of the hepatitis $B$ virus infection [49] and the tumor of esophagus [50]. Aflatoxins B1 (AFB1) is present mostly in the maize cereals and it caused many acute and chronic carcinoma, for instance due to the intensive exposure in the aflatoxins $\mathrm{B} 1$, its range up to $2 \mathrm{ppm}$, which caused the non-specific liver illness or liver carcinoma and its lead to death in few days. The rate of liver carcinoma is $50 \%$ higher than those people, which suffer from the liver disorder, hepatitis B, and hepatitis C. B1 aflatoxins caused the immunosuppressant and the nutritional deficiency due to the chronic effect of aflatoxins [51].

In addition of these, aflatoxins involved into the damage of protein synthesis, encouraged the coagulation, gaining of weight and the immune related disease [52]. Food borne aflatoxins are interfering the protein synthesis and activity of micronutrients such as vitamin A, C, K, E, B12, zine, selenium, calcium an iron [53]. On the other hand, feeding of animal may disturb, weight loss of the animals may occur in the domestic animals or even the death of animals that are survive on the crops and it contaminated through the aflatoxins [54]. Another effect which are caused by the aflatoxins in animals, i.e., lower the milk production, overcome the egg lying and suppressor the immune system of the animals because aflatoxins linked with the T-cells of the body (such as perforin, CD8+ T cells) and it is also involved to decrease the activity of vitamin $\mathrm{k}$. Humans are exposed to the aflatoxins by oral routes and they take the contaminated plants used as a food source such as maize and peanuts, and aflatoxins B1 which is obtained from these crops. From the animals, milk and meat, which are, obtain and these animals fed on the aflatoxins contaminated food, which have AFM1, human takes these aflatoxins through the plant and animal sources [55].

\section{Future Approaches of Aflatoxins}

The utilize of atoxigenic A. flavus for the competitive avoidance of toxigenic strains and the avoidance of AF contamination is solidified 
in a few countries around the world. The consistent follow-up for the advancement of microbial biopesticides infers directly from the mechanical approach of modern agribusiness. In other words, biopesticides, utilizing microorganisms as dynamic fixings, must be items reasonable for mechanical generation and likely to be utilized taking after practices that have already been created for chemical based plant security items. They truly represent one more tool accessible for agriculturists to decrease the manufactured chemical input in agricultural generation, much asked by all partners. Many biopesticides, herbicides and bio fungicides are used to control the contaminations, they have ability to control the contaminations.

Microorganisms that act with competition-based instruments require an intensive information of the connections that are built up between the diverse components included in an infection (plant, pathogen, and other biotic and abiotic natural factors). These microorganisms have ability to change the toxins into simple one because they have specialized enzymes to remove the toxins such as aflatoxins. Microorganism like bacteria, probiotic bacteria, fungi, yeast and many other types are mostly used to control the contamination of aflatoxins. In the future, there are many bacterial species are used to inhibit the growth of aflatoxins. New approach of transgenic foods and cereals are grown by the farmer because they have $90 \%$ less chances for the growth of aflatoxins contamination and they safeguard the food and feeds by transgenic seed and transgenic plants.

\section{Biosensors}

Biosensor and other different methods, which are used to detect the aflatoxins, very precisely, these techniques have multidisciplinary tools, which identify the aflatoxins and check the quantity and concentration of aflatoxins. Biosensors are used to detect the toxins and it work on the basis of immunochemical and physiochemical principles, such as electrochemical and DNA. Biosensors are widely used in the food field, food industries, healthcare and bio-defense [56]. Many types of biosensors are used to detect the aflatoxins. So that, these biosensors are combined with the other techniques such as, immunochemical methods. Biosensors are used to create the junction with the immunochemical methods. Junction created by the biosensors are based on the high affinity of antigen-antibodies interaction and reason to use biosensor is to enhance the sensitivity and lower the detection of aflatoxins [57].

\section{Antigen-antibodies detection methods}

Immunoassay techniques or methods, which are used to determine the immunochemical detection of the aflatoxins, in which the antigen-antibodies reaction (Ag-Ab) is carried out [58]. The method which is used to determine the aflatoxins on basis of antibodies-antigen interaction is ELISA (Enzyme-linked Immunosorbent Assay). It is very useful, accurate, very sensitive method to determine the aflatoxins. By using this method, antigen binds with the antibodies and determine the toxic compound present in the sample. Another technique is involved under the umbrella of immunoassay, which is used to qualitative and semi-qualitative determination of aflatoxins in the food and feed, crops, cereals and milk, it is known as LFIAs (lateral flow immunoassay) [59,60]. So that, antibodies-antigen binding techniques are unique and very useful to identify the aflatoxins.

\section{Proper drying process}

Drying temperature and the drying time of grains which may affects the on the development of the aflatoxins in the stored grains of maize. Slow drying with low heat for a long time may also cause the aflatoxins contaminations.

\section{Layer-in-bin drying process}

Drying maize in the deep layers can provide the conditions for the development of aflatoxins and other mold productions. The temperature for this drying process is 10 to 200 Farenheits above the outside conditions.

\section{Maintained storage conditions}

Proper storage is the important stage for prevent the aflatoxins contamination. Moisture content which is present in store grains is less than $12-13 \%$ to reduce the production of aflatoxins in the maize grain. Drying fans are also used for the drying of grain in stored tanks [61]. Proper storage has many benefits because of proper storage, mold attack, aflatoxins productions, insects and rodent attacks will reduce. Aerated grain, which is store at specific temperature and conditions. The storage place temperature is $100 \mathrm{~F}$ which is warmer than the outside air temperature and humidity is out $65 \%$. The proper air flow rate is $1 / 10$ cubic feet per bushel per minute, which is enough from for cooling the grain. The air which should be enter at the top of the bin and exhausted from the bottom of the bin, it is because of the whole moisture content which is removed from the grain. During the aeration process, never increase the heat. Grain which is present in storage must be check after 3 to 4 weeks. In storage, determined the insect activity, environmental conditions and fungus growth rate on the stored grains. Fungus species which may cause the production of aflatoxins present on the maize crop and its level is normally higher than the other crops.

\section{Ozone technique}

It is very sufficient to destroy the aflatoxins by an electrophilic attack on the double-bonded carbons (C8-C9) of the furan ring of aflatoxins resulting which is formation of the primary ozonide's followed by the rearrangement into the monozonide derivatives like organic acids, ketones and aldehydes, further detail which is linked to the ozone degradation, basically it is mechanism which is used for the aflatoxins B1 (AFB1) [62]. There are many applications of ozone, which involve to the aflatoxin degradation in the cereal, but it is less useful because this process needs high cost and not every agricultural producer used this method [63]. So that, another strategy which is used behind the degradation of aflatoxins known as gamma rays. The mechanism of gamma rays in which the free radicals produced when the radiolysis of the water and other units that attack to the terminal furan ring of aflatoxins B1 (AFB1) that resulting in the byproducts of diminish biological activity [64]. The effectiveness and efficiency of the gamma rays may enhance when it linked with the other techniques.

\section{Conclusion}

Aflatoxins are the toxins, mutagenic and carcinogenic compounds, which have ability to cause the acute and carcinogenic diseases such as liver carcinoma. Aflatoxins present in the food and feed, which give the harmful effects on human as well as the other living organisms. Aflatoxin's production is basically depended upon the fungus species named as Aspergillus flavus species. Aspergillus 
flavus grow on the cereals crops such as maize crops, rice crops and the nuts, which may cause the production of aflatoxins and the metabolites of aflatoxins for instance, AFB1, AFG1, AFM1, AFB2, AFG2 and the AFM2. Aflatoxins AFB1 produces from the cereals and crops and the AFM1 from the milk. The contamination occurred in whole field spread through the wind and insect body. Basically, many types of contamination occurred such as aflatoxins and many other mycotoxins which can be detect by the many biological techniques such as chromatography, HPLC, many immunochemical assay techniques such as ELISA and many other techniques are used to detect the aflatoxins and determined the quantity, quality and concentration of the aflatoxins. There are many methods to control the aflatoxins contamination in pre-harvest, post-harvest, harvest, during the processing and storage conditions. Lowering the moisture content, proper handling the grains, use of biopesticides and the environmental conditions are necessary to control the contamination of maize grains. There are many other compounds such as herbicides, pesticides, fungicides, plant extracts, oil from the different vegetables and fruits such as lemongrass, lemon and orange, many biological controls are used to inhibit the growth of aflatoxins. These biological controls such as probiotic bacteria, many fungi, yeast and algae used to prevent the growth, proliferations of fungal growth and diminish the activity of aflatoxins causes fungus known as Aspergillus flavus. These biological agents have antagonistic properties against the fungus, up to the $80-90 \%$ diminish the contamination from the crops. Many enzymes which are obtained from the microorganisms also play an important role to control the aflatoxins. So that, these are many methods and biological control which limit the growth of aflatoxins and safeguard the food and feed and save the lives of human as well as animals.

There are following methods to identify the aflatoxins, for instance chromatography i.e., Gas chromatography (GC), Liquid Chromatography (LC), thin layer chromatography (TLC), Enzymelinked immunosorbent assay (ELISA), radioimmunoassay (RIA), immunoaffinity column assay (ICA), the mass spectrometry imaging (MSI), biosensor and also other methods are used. By quantifying the aflatoxins, the common and accurate method is used, known as chromatography. For first analysis of the aflatoxins, gas chromatography (GC) is used to detect and quantify the aflatoxins. Due to the advancement in technology, another chromatography techniques known as, Liquid Chromatography (LC) and the Thin Layer Liquid Chromatography (TLC) are used for the sensitive detection of aflatoxins in crops, cereals and grains. Other important type of the chromatography is HPLC (High Performance Liquid Chromatography) [65]. By using the HPLC, easily checked out the type of aflatoxins either the AFB1 and AFM1 or its also used to check the metabolites of AFB1 and other toxins [66]. HPLC detects the maximum amount of the aflatoxins and mycotoxins and other fungal contamination in the given sample. HPLC linked with the mass spectroscopy because they give precise and very sensitive to give the exact concentration of aflatoxins and other compounds identify in a single attempt. Another technique used which is linked with the HPLC for the detection is fluorescence spectroscopy, that is used for the basic types of aflatoxins. Fluorescence spectroscopy used in the HPLC as well as the Thin Layer Chromatography (TLC). So that, there are many combined methods for the detection of aflatoxins in a liquid solution, which are given the best and accurate results. For instance, in the immunoaffinity column sample, which is clean up, and it is follow by the normal or reverse stage of the HPLC separation with the fluorescence detection.

\section{References}

1. Ito $\mathrm{Y}$, et al. "Aspergillus pseudotamarii, a new aflatoxin producing species in Aspergillus section Flavi." Mycological Research. 2001; 105: 233-239.

2. Wicklow DT, et al. "Effect of intraspecific competition by Aspergillus flavus on aflatoxin formation in suspended disc culture." Mycological Research. 2003; 107: 617-623.

3. Achaglinkame MA, et al. "Aflatoxin contamination in cereals and legumes to reconsider usage as complementary food ingredients for Ghanaian infants: A review." Journal of Nutrition \& Intermediary Metabolism. 2017; 10: 1-7.

4. Yu J, et al. "Clustered pathway genes in aflatoxin biosynthesis." Applied and environmental microbiology. 2004; 70: 1253-1262.

5. Cambaza E, et al. "A glance at aflatoxin research in Mozambique." International journal of environmental research and public health. 2018; 15 : 1673.

6. Liu Y and F Wu. "Global burden of aflatoxin-induced hepatocellular carcinoma: a risk assessment." Environmental health perspectives. 2010; 118: 818-824.

7. Wu F. "Global impacts of aflatoxin in maize: trade and human health." World Mycotoxin Journal. 2015; 8: 137-142.

8. Dors GC, et al. "Aflatoxins: contamination, analysis and control." Embrapa Suínos e AvesCapítulo em livro científico (ALICE). 2011.

9. Masinde LA, et al. "Colloidal gold based immunochromatographic strip for the simple and sensitive determination of aflatoxin B1 and B2 in corn and rice." Microchimica Acta. 2013; 180: 921-928.

10. Karaaslan M and Y Arslanğray. "Aflatoxins B1, B2, G1, and G2 contamination in ground red peppers commercialized in Sanliurfa, Turkey." Environmental monitoring and assessment. 2015; 187: 184.

11. Hussein HS and JM Brasel. "Toxicity, metabolism, and impact of mycotoxins on humans and animals." Toxicology. 2001; 167: 101-134

12. Xu Q, et al. "Critical role of caveolin-1 in aflatoxin B1-induced hepatotoxicity via the regulation of oxidation and autophagy." Cell Death \& Disease. 2020; 11: 1-16.

13. Taghizadeh SF, et al. "Occurrence of mycotoxins in rice consumed by Iranians: a probabilistic assessment of risk to health." Food Additives \& Contaminants: Part A. 2020; 37: 342-354.

14. Pour $\mathrm{SH}$, et al. "Aflatoxin $\mathrm{M}_{1}$ contamination level in Iranian milk and dairy products: A systematic review and meta-analysis." World Mycotoxin Journal. 2020; 13: 67-82.

15. Bbosa GS, et al. "Review of the biological and health effects of aflatoxins on body organs and body systems." Aflatoxins-recent advances and future prospects. 2013; 12: 239-265.

16. Klvana $\mathrm{M}$ and $\mathrm{U}$ Bren. "Aflatoxin B1-formamidopyrimidine DNA adducts: Relationships between structures, free energies, and melting temperatures." Molecules. 2019; 24: 150.

17. Williams $\mathrm{JH}$, et al. "Human aflatoxicosis in developing countries: a review of toxicology, exposure, potential health consequences, and interventions." The American journal of clinical nutrition. 2004; 80: 1106-1122.

18. Turner PC. "The molecular epidemiology of chronic aflatoxin driven impaired child growth.” Scientifica. 2013.

19. Joint $F$, et al. Evaluation of certain contaminants in food: eighty-third report of the Joint FAO/WHO Expert Committee on Food Additives. World Health Organization.

20. Amare MG and NP Keller. "Molecular mechanisms of Aspergillus flavus secondary metabolism and development." Fungal Genetics and Biology. 2014; 66 : 11-18. 
21. Cole RJ, et al. Handbook of secondary fungal metabolites. 2003; 3.

22. Mitchell NJ, et al. "Potential economic losses to the US corn industry from aflatoxin contamination." Food Additives \& Contaminants: Part A. 2016; 33 $540-550$

23. Heshmati A, et al. "Co-occurrence of aflatoxins and ochratoxin A in dried fruits in Iran: Dietary exposure risk assessment." Food and Chemical Toxicology 2017; 106: 202-208.

24. Abrunhosa L, et al. "A review of mycotoxins in food and feed products in Portugal and estimation of probable daily intakes." Critical reviews in food science and nutrition. 2016; 56: 249-265.

25. Xia J, et al. "Simultaneous determination of melamine and related compounds by capillary zone electrophoresis." Food Control. 2010; 21: 912-918.

26. Garcia-Cela E, et al. "Carbon dioxide production as an indicator of Aspergillus flavus colonisation and aflatoxins/cyclopiazonic acid contamination in shelled peanuts stored under different interacting abiotic factors." Fungal Biology. 2020; 124: $1-7$

27. Ito $Y$, et al. "Aspergillus pseudotamarii, a new aflatoxin producing species in Aspergillus section Flavi." Mycological Research. 2001; 105: 233-239.

28. Peterson SW, et al. "Aspergillus bombycis, a new aflatoxigenic species and genetic variation in its sibling species, A. nomius." Mycologia. 2001; 93: 689 703

29. Frisvad JC, et al. "Emericella astellata, a new producer of aflatoxin B1, B2 and sterigmatocystin." Letters in applied microbiology. 2004; 38: 440-445.

30. Muhialdin BJ, et al. "Review on the Biological Detoxification of Mycotoxins Using Lactic Acid Bacteria to Enhance the Sustainability of Foods Supply." Molecules. 2020; 25: 2655

31. Okafor S and A Eni. "Microbial Quality and the Occurrence of Aflatoxins in Plantain/Yam and Wheat Flours in Ado-Odo Ota." E\&ES. 2018.

32. Bennett J and M Klich. "Mycotoxins. Clinical Microbiological Reviews. 2003; 16.

33. Heilmann C and P Grandjean. Immunotoxicity: Impacts and Research Approaches. Health Impacts of Developmental Exposure to Environmenta Chemicals, Springer. 2020; 175-190.

34. Chun HS, et al. "Determination of aflatoxin levels in nuts and their products consumed in South Korea." Food Chemistry. 2007; 102: 385-391.

35. Wacoo AP, et al. "Methods for detection of aflatoxins in agricultural food crops." J. appl. Chem. 2014: 706291.

36. Meeting J. F. W. E. C. O. F. A. and W. H. Organization. Safety evaluation of certain food additives and contaminants, World Health Organization. 2014

37. Udovicki B, et al. "Overview on the Mycotoxins Incidence in Serbia in the Period 2004-2016." Toxins. 2018; 10: 279

38. Mahato DK, et al. "Aflatoxins in food and feed: an overview on prevalence, detection and control strategies." Frontiers in Microbiology. 2019; 10: 2266.

39. Nordkvist E, et al. "Aflatoxin contamination of consumer milk caused by contaminated rice by products in compound cattle feed." Journal of the Science of Food and Agriculture. 2009; 89: 359-361.

40. Maigua IRS, et al. "Evaluación de la contaminación por aflatoxinas B1, B2, G1 y G2 en maíz amarillo duro.” Revista ESPAMCIENCIA. 2018; 9: 13-21.

41. Reiter EV, et al. "Aflatoxins in rice-a limited survey of products marketed in Austria." Food Control. 2010; 21: 988-991.

42. Marasas WF, et al. "Mycotoxins: a global problem." Mycotoxins: Detection Methods, Management, Public Health and Agricultural Trade. $4^{\text {th }}$ edition. Oxford, UK: CABI. 2008: 29-39.

43. Achaglinkame MA, et al. "Aflatoxin contamination in cereals and legumes to reconsider usage as complementary food ingredients for Ghanaian infants: A review." Journal of Nutrition \& Intermediary Metabolism. 2017; 10: 1-7.

44. Lewis L, et al. "Aflatoxin contamination of commercial maize products during an outbreak of acute aflatoxicosis in eastern and central Kenya."
Environmental health perspectives. 2005; 113: 1763-1767.

45. Medina A, et al. "Climate change, food security and mycotoxins: do we know enough?" Fungal Biology Reviews. 2017; 31: 143-154.

46. Sekiyama BL, et al. "Aflatoxins, ochratoxin $A$ and zearalenone in maizebased food products." Brazilian Journal of Microbiology. 2005; 36: 289-294.

47. Chauhan NM, et al. "Fungal infection and aflatoxin contamination in maize collected from Gedeo zone, Ethiopia." Springer Plus. 2016; 5: 753

48. Mehmood A, et al. "In vitro production of IAA by endophytic fungus Aspergillus awamori and its growth promoting activities in Zea mays." Symbiosis. 2019; 77: $225-235$

49. Yu J, et al. "Aspergillus flavus genomics: gateway to human and animal health, food safety, and crop resistance to diseases." Revista iberoamericana de micología. 2005; 22: 194-202.

50. Scheidegger $\mathrm{K}$ and $\mathrm{G}$ Payne. "Unlocking the secrets behind secondary metabolism: a review of Aspergillus flavus from pathogenicity to functional genomics." Journal of Toxicology: Toxin Reviews. 2003; 22: 423-459.

51. Afum $C$, et al. "Association between aflatoxin $M_{1}$ and liver disease in HBV/ $\mathrm{HCV}$ infected persons in Ghana." International journal of environmental research and public health. 2016; 13: 377

52. Abbas $\mathrm{H}$, et al. "Spatial variability of Aspergillus flavus soil populations unde different crops and corn grain colonization and aflatoxins." Canadian Journal of Botany. 2004; 82: 1768-1775.

53. Wang X-Z, et al. "Genetic polymorphisms in ataxin-3 and liver cirrhosis risk related to aflatoxin B1." Oncotarget. 2018; 9: 27321.

54. Wild $C$ and $P$ Turner. "The toxicology of aflatoxins as a basis for public health decisions." Mutagenesis. 2002; 17: 471-481.

55. Pinhão $M$, et al. Mixtures health effects: mycotoxins in food. $1^{\text {st }}$ Workshop on Human Biomonitoring in Portugal" Bridging Chemical Exposure to Human Health", INSA. 2018

56. Ho J-A and R Wauchope. "A strip liposome immunoassay for aflatoxin B1." Analytical chemistry. 2002; 74: 1493-1496.

57. Anfossi L, et al. "Lateral flow immunoassays for aflatoxins $B$ and $G$ and for aflatoxin M1." Aflatoxins-Recent Adv. Future Prospect. 2013: 315-339.

58. Donkor O, et al. "Techniques 8 and Control for of Mycotoxins Detection, Quantification in Dairy Products." Microbial Toxins in Dairy Products. 2016 7; 201

59. Muscarella M, et al. "Validation according to European Commission Decision 2002/657/EC of a confirmatory method for aflatoxin $M_{1}$ in milk based on immunoaffinity columns and high performance liquid chromatography with fluorescence detection." Analytica chimica acta. 2007; 594: 257-264.

60. Chauhan NM, et al. "Fungal infection and aflatoxin contamination in maize collected from Gedeo zone, Ethiopia." SpringerPlus. 2016; 5: 753.

61. Kaaya AN and W Kyamuhangire. "The effect of storage time and agroecological zone on mould incidence and aflatoxin contamination of maize from traders in Uganda." International journal of food microbiology. 2006; 110: 217-223.

62. Diao $\mathrm{E}$, et al. "Ozonolysis mechanism and influencing factors of aflatoxin B1: A review." Trends in Food Science \& Technology. 2013; 33: 21-26.

63. Womack ED, et al. "A recent review of non-biological remediation of aflatoxin contaminated crops." Journal of the Science of Food and Agriculture. 2014; 94: $1706-1714$

64. Vicente $S$, et al. "Aflatoxins distribution in fractions derived from tofu production." Food Additives \& Contaminants: Part A. 2019; 36: 1559-1566.

65. Reinholds I, et al. "Recent applications of carbonaceous nano sorbents for the analysis of mycotoxins in food by liquid chromatography: a short review." World Mycotoxin Journal. 2019; 12: 31- 43

66. Singh S, et al. "An overview of challenges and elimination of aflatoxin contamination in maize (Zea mays)." Journal of Pharmacognosy and Phytochemistry. 2019; 8: 2895-2906. 